\title{
Change in the risk stratification of prostate cancer after Slide Review by a uropathologist: the experience of a reference center for the treatment of prostate cancer
}

George Camara-Lopes², Gustavo Nader Marta', Elton Trigo Teixeira Leite', Gabriela Silva Moreira de Siqueira' ${ }^{1}$, Samir Abdallah Hanna', João Luis Fernandes da Silva', L. H. Camara-Lopes², Katia R. M. Leite ${ }^{3}$

${ }^{1}$ Department of Radiotherapy and ${ }^{2}$ Laboratory of Surgical Pathology, Sirio Libanes Hospital, Sao Paulo and ${ }^{3}$ Laboratory of Medical Research, Department of Urology, University of Sao Paulo Medical School LIM55, Sao Paulo, Brazil

\section{ABSTRACT}

Introduction: Brachytherapy is an option for treating low-risk prostate cancer (PC). Biochemical control of low-risk disease can reach 95\%. The practice advocated is that a review of prostate biopsies should be mandatory before choosing the best treatment for patients with PC. Our objective was to evaluate the change in PC risk after review of a prostate biopsy by an experienced uropathologist at a reference hospital.

Materials and Methods: Between December 2003 and August 2012, 182 men were referred to our institution for brachytherapy to treat PC. Their slides were reviewed by the same uropathologist.

Results and Discussion: Classification risk disagreement occurred in 71 (39\%) cases, including one in which no tumor was observed. The main cause of risk change was related to the Gleason score (GS), with 57 (81.4\%) cases upgraded to GS 7 or 8. Tumor volume was also compared, although only the number of fragments was reported in most original reports. The concordance of the number of cores affected by tumor was $43.9 \%$, and in $49 \%$ of the cases, the number was decreased by the uropathologist. Perineural invasion (PNI) was reported in one quarter of original reports, and the agreement was 58\%.

Conclusion: Slide review by an uropathologist remains essential at reference radiotherapy centers for the treatment of PC. The change in PC risk evaluation is mainly due to the GS, but tumor volume and PNI, which are important for the characterization of tumor aggressiveness, are also misinterpreted and could drive a change in the therapy choice.

\section{ARTICLE INFO}

\section{Key words:}

Prostatic Neoplasms;

Brachytherapy; Androgens

Int Braz J Urol. 2014; 40: 454-62

Submitted for publication: October 10, 2013

\section{Accepted after revision:}

March 26, 2014

\section{INTRODUCTION}

Currently, the management of prostate cancer $(\mathrm{PC})$ varies from active surveillance to palliative androgen blockage, depending on the prognostic risk stratification, whose determinants are the Gleason score (GS), PSA serum levels and the clinical stage. Among these determinants, the
GS is the most important defining factor in PC aggressiveness $(1,2)$. The discrepancies in grading GSs among observers can reach up to 64\%, even among experienced uropathologists (3-9). This concern has been further confirmed since the 2005 International Society of Urological Pathology (ISUP)'s modification of Gleason grading (10). Although only 50\% of American hospitals adopt 
this practice (11), a second pathology review is currently recommended as routine practice for patients referred to specialized centers for PC treatment before the definition of a better management protocol (12-14).

Patients with low-risk PC can be treated with brachytherapy, and the reported biochemical control is as high as 95\% (15). Inaccuracy in risk stratification and treatment selection could compromise curability rates, affecting patients' quality of life and increasing the expenses for the health system (16). The objective of this study was to evaluate the rates of disagreement in the evaluation of prostate needle biopsies after a slide review by an experienced uropathologist at a reference center in treating PC.

\section{MATERIALS AND METHODS}

In total, 182 men were referred to our radiotherapy department, which is specialized in treating PC, from December 2003 to August 2012. The median age of the patients was 64 years old, with a range of 45 to 84 years. The mean PSA level was $6.4 \mathrm{ng} / \mathrm{mL}$ (SD $3.2 \mathrm{ng} / \mathrm{mL}$ ). The clinical stage was T1c for $126(69.2 \%)$ and T2a,b for the remaining patients. Eighty-nine (48.9\%) patients were in the low-risk group, 81 (44.5\%) were in the intermediate-risk group, and 12 (6.6\%) were in the high-risk group.

Hematoxylin and eosin-stained slides were reviewed by the same uropathologist. The median number of biopsy cores was 12 , with a range of 2 to 36. Several parameters from the initial diagnosis, such as the GS, the number of fragments that were positive for tumor, the percentage of tumor in the most affected biopsy core and the total percentage of tumor in all cores, and the presence of perineural invasion (PNI) were compared with the values in the final reviewed report. A major discrepancy was defined as a difference that directly affected the patient's risk stratification.

The risk assessment was made based on the classification developed by D'Amico et al. $(17,18)$. Briefly, low risk was defined as PSA $\leq 10 \mathrm{ng} / \mathrm{mL}$, $\mathrm{GS} \leq 6$ and a clinical stage of T1-2a; intermediate risk, PSA $>10$ but $\leq 20 \mathrm{ng} / \mathrm{mL}, \mathrm{GS}=7$ and a clinical stage of T2b; and high risk, PSA > 20ng/
mL, GS $>8$ or a clinical stage of T2c or T3a. This system does not take into consideration other parameters observed on the slides review such as PNI and tumor volume reflected by the percentage of tumor extend. However, these additional findings added to the study are important for radiotherapists to tailor the better treatment. PNI is related to extraprostatic extension of tumor and has been a matter of concern for brachytherapy.

This project was approved by the Sirio Libanes Ethical Board (51741).

\section{RESULTS}

Significant discrepancies that could affect risk stratification occurred in 58 (31.9\%) cases. In 1 (0.55\%), no tumor was observed, and in 57 $(81.4 \%)$ cases, the risk was elevated. The main change in risk stratification was due to discordance in the GS. The results are detailed in Table-1. Tumors previously diagnosed as low grade (GS $\leq$ 6) were upgraded to 7 (53-93\%) or $8(4-7 \%)$. In total, $42(23 \%)$ cases were previously diagnosed as $\mathrm{GS} \geq 7$, with 13 (30.1\%) downgraded to GS $\leq 6$.

In 157 original reports, only the number of cores that were positive for tumor informed the tumor volume. Considering this feature, concordance occurred in $69(43.9 \%)$ cases. In 43 (27.3\%), the number of positive cores was underestimated (Table-2).

PNI is another important phenomenon related to extraprostatic extension and should be mentioned in final reports $(19,20)$. This information was present only in $47(25.8 \%)$ reports. PNI was reported as positive in 12 original reports, and the review agreed in 7 (58.3\%) cases. There were $24(13.1 \%)$ cases positive for PNI after the slides review. In two cases, PNI was designated as negative while the review considered PNI as positive.

\section{DISCUSSION}

Brachytherapy is a good option for treating low- and intermediate-risk prostate adenocarcinoma, with good results for disease control and lower morbidity associated with urinary incontinence and sexual dysfunction $(21,22)$. Prostate biopsy is the only method for diagnosing PC, 
Table 1 - Frequency in distribution of Gleason scores on prostate biopsy.

\begin{tabular}{lcccccccc}
\hline \multicolumn{1}{c}{ Reviewed Gleason score } \\
\hline GS Original & $5(2+3)$ & $5(3+2)$ & $6(3+3)$ & $7(3+4)$ & $7(4+3)$ & $8(4+4)$ & No tumor & Total \\
\hline $4(2+2)$ & 0 & 1 & 3 & 2 & 1 & 0 & 0 & 7 \\
$5(2+3)$ & 1 & 0 & 2 & 1 & 0 & 0 & 0 & 4 \\
$5(3+2)$ & 1 & 2 & 5 & 1 & 2 & 1 & 0 & 12 \\
$6(3+3)$ & 4 & 5 & 58 & 31 & 15 & 3 & 1 & 117 \\
$7(3+4)$ & 0 & 0 & 9 & 6 & 7 & 5 & 0 & 27 \\
$7(4+3)$ & 0 & 0 & 2 & 2 & 5 & 1 & 0 & 10 \\
$8(4+4)$ & 0 & 1 & 1 & 0 & 1 & 1 & 0 & 4 \\
$8(5+3)$ & 0 & 0 & 0 & 0 & 0 & 1 & 0 & 1 \\
\hline Total & 6 & 9 & 80 & 43 & 31 & 12 & 1 & 182 \\
\hline
\end{tabular}

Table 2 - Number of cores positive for tumors. Comparison between the first and the review evaluation.

\begin{tabular}{lccccccccccc}
\hline \multicolumn{1}{c}{ Review report } \\
\hline Original report & 0 & 1 & 2 & 3 & 4 & 5 & 6 & 7 & 8 & $9-12$ & Total \\
\hline 1 & 0 & 25 & 9 & 3 & 3 & 1 & 0 & 0 & 0 & 0 & 41 \\
2 & 0 & 4 & 27 & 5 & 3 & 2 & 0 & 0 & 0 & 1 & 42 \\
3 & 1 & 1 & 2 & 7 & 5 & 1 & 0 & 2 & 0 & 0 & 19 \\
4 & 0 & 1 & 3 & 7 & 6 & 1 & 0 & 1 & 0 & 0 & 19 \\
5 & 0 & 0 & 0 & 3 & 2 & 3 & 1 & 1 & 0 & 0 & 10 \\
6 & 0 & 0 & 2 & 0 & 6 & 4 & 1 & 1 & 2 & 0 & 16 \\
7 & 0 & 0 & 0 & 1 & 1 & 0 & 1 & 0 & 0 & 1 & 4 \\
8 & 0 & 0 & 0 & 0 & 0 & 1 & 0 & 0 & 0 & 0 & 1 \\
$9-12$ & 0 & 0 & 0 & 0 & 4 & 0 & 0 & 0 & 1 & 0 & 5 \\
\hline Total & 1 & 31 & 43 & 26 & 30 & 13 & 3 & 5 & 3 & 2 & 157 \\
\hline
\end{tabular}

and the evaluation of tumor characteristics has a crucial role in the process of clinical decision-making, with the GS being the most important prognostic factor. However, there is high variability in interobserver interpretation of GSs, even among experienced uropathologists. A recent report described a kappa value of $0.57(95 \% \mathrm{CI})$ for GS evaluation between two uropathologists, exemplifying the subjectivity of this classification
(23). This discordance could lead to the choice of a non-ideal treatment, with a large impact on disease control, side effects and financial expenses for the health system. Therefore, slide review by an uropathologist at reference hospitals for PC treatment is currently recommended $(12,24)$.

In this study, we described the review of prostate biopsies from patients referred to a reference center for brachytherapy treatment. We 
found that risk stratification changed in one third of patients, including $1(0.55 \%)$ case in which no tumor was observed. A large study described the absence of a tumor in 1.2\% of cases after expert review and considered a second opinion by an uropathologist as mandatory when facing a PC diagnosis (12).

The main discordance that we found was an underestimation of the GS responsible for changing the risk stratification. A report from Sooriakumaran et al. described 19\% disagreement that was mainly related to risk underestimation (25). Kishimoto et al. described numbers similar to ours, reporting 38\% discordance, of which 82\% was related to underestimation of the GS (26).

The quantification of tumors is another important feature for defining tumor significance, and the number of cores that are positive for tumor, the amount of tumor in a single core and the total percentage of tumor in all cores are important data that should be mentioned in final reports (27-29). The tumor volume was cited in 157 (86.3\%) final reports, although only the number of positive cores was described. There was concordance in the number of positive cores in nearly $44 \%$ of the cases. Curiously, the main discordance was the overestimation of positive cores by the general pathologist (51\%), including a case in which an uropathologist did not find cancer (0.6\%). The first report analyzing discrepancies in tumor volume was published by Brimo et al. in 2010 (12). Disagreement in the number of fragments that were positive for tumors occurred in 9\% of the cases, with the majority of cases (63\%) underestimated by the general pathologist.

After 2005 all cases were signed out according to the 2005 ISUP's consensus conference on Gleason grading for prostate cancer (10). Although we are not aware of the knowledge of the first examiner about the ISUP's recommendations we found some interesting results that are worth to be mentioned. There were $23(12.6 \%)$ original cases previously signed as GS $<6$, being 7 (30.4\%) classified as GS $4(2+2)$, all of these were upstaged after the review. The 2005 ISUP consensus considered that GS 4 is a very rare finding in prostate biopsies, most frequently present in transitional zone. Even GS 5 has been diagnosed in less than $1 \%$ of the cases.

Forty-six cases (25.2\%) were upstaged after the review. Since the 2005 ISUP's consensus several studies documented a shift towards assigning higher GS on both biopsy and radical prostatectomy specimens (30). From the upstaged cases after the review, $28(60.8 \%)$ had the secondary Gleason pattern upgraded, similar to other published.

Another interesting aspect that we reviewed in this study was information about PNI by tumors. The literature confirms that PNI is related to extraprostatic extension and should be affirmed or denied in biopsy reports $(19,20)$. PNI was mentioned in only $26 \%$ of original reports, and agreement occurred in 58\% of the cases. Although PNI is agreed to be an important factor related to aggressive pathologic features, it remains a con-

Figure 1 - Examples of biopsies that resulted in change in the final diagnosis. A) A case of atypical adenomatous hyperplasia or adenosis previously diagnosed as adenocarcinoma. B) Gleason pattern 4 in a case previously diagnosed as Gleason score $6(3+3)$. C) Perineural invasion (arrow) that had not been recorded in the original report.

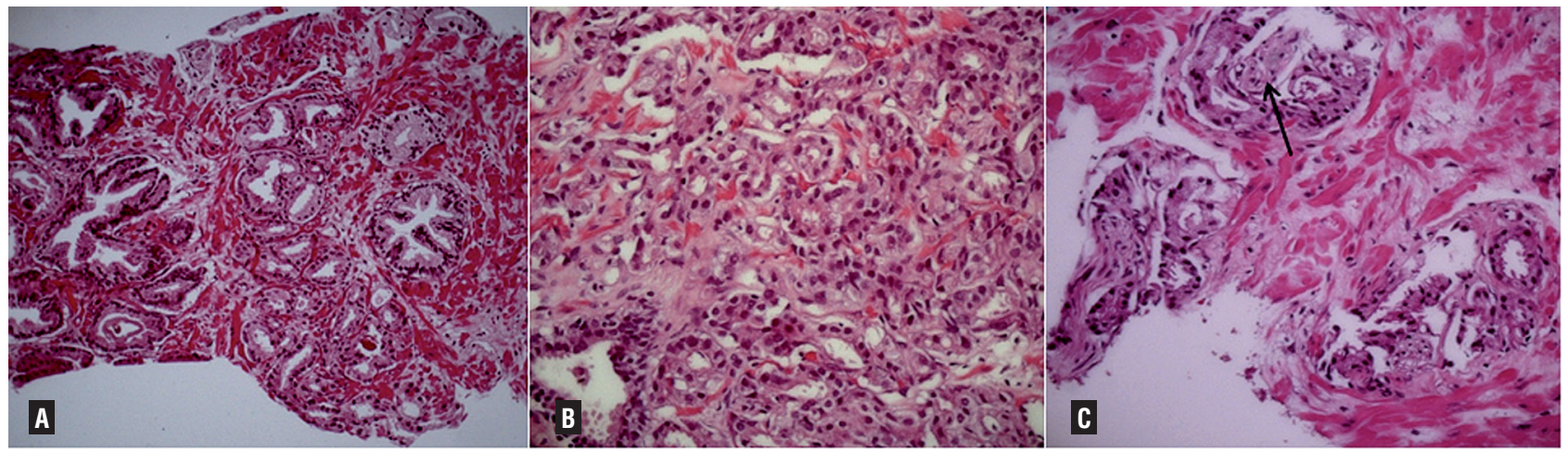


troversial issue in prostate pathology and a subject of interest since the mid 90's. In some studies the correlation between PNI and advanced stages is not confirmed in multivariate analysis. Billis et al. (31) found that PNI predicts higher tumor stages on radical prostatectomies only in univariate analysis. However, a study published by Loeb et al. (32) describes a correlation of PNI with EPE and seminal vesicle invasion also in multivariate analysis.

In conclusion, our results show that a second opinion on a prostate biopsy by an uropathologist, as part of a team devoted to the treatment of PC through the best characterization of all aspects related to disease risk, is essential for the choice of the best management protocol for better disease control, fewer side effects and fewer financial expenses.

\section{CONFLICT OF INTEREST}

None declared.

\section{REFERENCES}

1. D’Amico AV, Schultz D, Schneider L, Hurwitz M, Kantoff PW, Richie JP: Comparing prostate specific antigen outcomes after different types of radiotherapy management of clinically localized prostate cancer highlights the importance of controlling for established prognostic factors. J Urol. 2000; 163: 1797-801.

2. Blute ML, Bergstralh EJ, Partin AW, Walsh PC, Kattan MW, Scardino PT, et al.: Validation of Partin tables for predicting pathological stage of clinically localized prostate cancer. J Urol. 2000; 164: 1591-5.

3. Glaessgen A, Hamberg H, Pihl CG, Sundelin B, Nilsson B, Egevad L: Interobserver reproducibility of percent Gleason grade 4/5 in prostate biopsies. J Urol. 2004; 171: 664-7.

4. Mikami Y, Manabe T, Epstein JI, Shiraishi T, Furusato M, Tsuzuki T, et al.: Accuracy of gleason grading by practicing pathologists and the impact of education on improving agreement. Hum Pathol. 2003; 34: 658-65.

5. Renshaw AA, Schultz D, Cote K, Loffredo M, Ziemba DE, D'Amico AV: Accurate Gleason grading of prostatic adenocarcinoma in prostate needle biopsies by general pathologists. Arch Pathol Lab Med. 2003; 127: 1007-8.

6. Epstein $\mathrm{Jl}$ : Gleason score 2-4 adenocarcinoma of the prostate on needle biopsy: a diagnosis that should not be made. Am J Surg Pathol. 2000; 24: 477-8.
7. Bostwick DG: Gleason grading of prostatic needle biopsies. Correlation with grade in 316 matched prostatectomies. Am J Surg Pathol. 1994; 18: 796-803.

8. Ozdamar SO, Sarikaya S, Yildiz L, Atilla MK, Kandemir B, Yildiz S: Intraobserver and interobserver reproducibility of WHO and Gleason histologic grading systems in prostatic adenocarcinomas. Int Urol Nephrol. 1996; 28: 73-7.

9. Moreira Leite KR, Camara-Lopes LH, Dall'Oglio MF, Cury J, Antunes AA, Sañudo A, et al.: Upgrading the Gleason score in extended prostate biopsy: implications for treatment choice. Int J Radiat Oncol Biol Phys. 2009; 73: 353-6.

10. Epstein JI, Allsbrook WC Jr, Amin MB, Egevad LL; ISUP Grading Committee: The 2005 International Society of Urological Pathology (ISUP) Consensus Conference on Gleason Grading of Prostatic Carcinoma. Am J Surg Pathol. 2005; 29: 1228-42.

11. Gupta D, Layfield LJ: Prevalence of inter-institutional anatomic pathology slide review: a survey of current practice. Am J Surg Pathol. 2000; 24: 280-4.

12. Brimo F, Schultz L, Epstein Jl: The value of mandatory second opinion pathology review of prostate needle biopsy interpretation before radical prostatectomy. J Urol. 2010; 184: 126-30.

13. Bottke D, Golz R, Störkel S, Hinke A, Siegmann A, Hertle L, et al.: Phase 3 study of adjuvant radiotherapy versus wait and see in pT3 prostate cancer: impact of pathology review on analysis. Eur Urol. 2013; 64: 193-8.

14. D’Souza N, Loblaw DA, Mamedov A, Sugar L, Holden L: Prostate cancer pathology audits: is central pathology review still warranted? Can J Urol. 2012; 19: 6256-60.

15. Crook J: The role of brachytherapy in the definitive management of prostate cancer. Cancer Radiother. 2011; 15: $230-7$.

16. Wurzer JC, Al-Saleem TI, Hanlon AL, Freedman GM, Patchefsky A, Hanks GE: Histopathologic review of prostate biopsies from patients referred to a comprehensive cancer center: correlation of pathologic findings, analysis of cost, and impact on treatment. Cancer. 1998; 83: 753-9.

17. D’Amico AV, Moul J, Carroll PR, Sun L, Lubeck D, Chen $\mathrm{MH}$ : Cancer-specific mortality after surgery or radiation for patients with clinically localized prostate cancer managed during the prostate-specific antigen era. J Clin Oncol. 2003; 21: 2163-72.

18. Albertsen PC, Hanley JA, Gleason DF, Barry MJ: Competing risk analysis of men aged 55 to 74 years at diagnosis managed conservatively for clinically localized prostate cancer. JAMA. 1998; 280: 975-80.

19. Katz B, Srougi M, Dall'Oglio M, Nesrallah AJ, Sant'anna AC, Pontes $\mathrm{J} \mathrm{Jr}$, et al.: Perineural invasion detection in prostate biopsy is related to recurrence-free survival in patients submitted to radical prostatectomy. Urol Oncol. 2013; 31: 175-9. 
20. Cozzi G, Rocco BM, Grasso A, Rosso M, Abed El Rahman D, Oliva I, et al.: Perineural invasion as a predictor of extraprostatic extension of prostate cancer: a systematic review and metaanalysis. Scand J Urol. 2013; 47: 443-8.

21. D'Amico AV, Whittington R, Malkowicz SB, Schultz D, Blank $\mathrm{K}$, Broderick GA, et al.: Biochemical outcome after radical prostatectomy, external beam radiation therapy, or interstitial radiation therapy for clinically localized prostate cancer. JAMA. 1998; 280: 969-74.

22. Grimm P, Billiet I, Bostwick D, Dicker AP, Frank S, Immerzeel $\mathrm{J}$, et al.: Comparative analysis of prostate-specific antigen free survival outcomes for patients with low, intermediate and high risk prostate cancer treatment by radical therapy. Results from the Prostate Cancer Results Study Group. BJU Int. 2012; 109(Suppl 1): 22-9.

23. Goodman M, Ward KC, Osunkoya AO, Datta MW, Luthringer D, Young AN, et al.: Frequency and determinants of disagreement and error in gleason scores: a population-based study of prostate cancer. Prostate. 2012; 72: 1389-98.

24. Epstein JI, Walsh PC, Sanfilippo F: Clinical and cost impact of second-opinion pathology. Review of prostate biopsies prior to radical prostatectomy. Am J Surg Pathol. 1996; 20: 851-7.

25. Sooriakumaran P, Lovell DP, Henderson A, Denham P, Langley SE, Laing RW: Gleason scoring varies among pathologists and this affects clinical risk in patients with prostate cancer. Clin Oncol (R Coll Radiol). 2005; 17: 655-8.

26. Kishimoto R, Saika T, Bekku K, Nose H, Abarzua F, Kobayashi Y, et al.: The clinical impact of pathological review on selection the treatment modality for localized prostate cancer in candidates for brachytherapy monotherapy. World J Urol. 2012; 30: 375-8.

27. Cheng L, Poulos CK, Pan CX, Jones TD, Daggy JK, Eble JN, et al.: Preoperative prediction of small volume cancer (less than $0.5 \mathrm{ml}$ ) in radical prostatectomy specimens. J Urol. 2005; 174: 898-902.
28. Katz B, Srougi M, Camara-Lopes LH, Antunes AA, Nesrallah $L$, Nesrallah $A$, et al.: The accuracy of pathological data for the prediction of insignificant prostate cancer. Int Braz J Urol. 2012; 38: $760-8$.

29. Antunes AA, Srougi M, Dall'Oglio MF, Crippa A, Nesrallah AJ, Nesrallah LJ, et al.: Preoperative determination of prostate cancer tumor volume: analysis through biopsy fragments. Int Braz J Urol. 2007; 33: 477-83; discussion 484-5.

30. Billis A, Guimaraes MS, Freitas LL, Meirelles L, Magna LA, Ferreira U: The impact of the 2005 international society of urological pathology consensus conference on standard Gleason grading of prostatic carcinoma in needle biopsies. J Urol. 2008; 180: 548-52; discussion 552-3.

31. Billis A, de Quintal MM, Meirelles L, Freitas LL, Magna LA, Ferreira U: Does tumor extent on needle prostatic biopsies influence the value of perineural invasion to predict pathologic stage \&gt; T2 in radical prostatectomies? Int Braz J Urol. 2010; 36: 439-47; discussion 448, 448-9.

32. Loeb S, Epstein JI, Humphreys EB, Walsh PC: Does perineural invasion on prostate biopsy predict adverse prostatectomy outcomes? BJU Int. 2010; 105: 1510-3.
Correspondence address:

George Camara-Lopes, MD

Department of Radiotherapy, Sirio Libanes Hospital, Sao Paulo Av. Angélica, 2318, 5 andar, Consolação Sao Paulo, SP, 01228-000, Brazil E-mail: camaralopes@gmail.com 


\section{EDITORIAL COMMENT}

The referred manuscript is a large sample study and demonstrated good results in terms of low overall incidence of symptomatic urinary tract infection. These results denoted that ciprofloxacin still was effective in promoting antibiotic prophylaxis in the population evaluated despite the current increase in bacterial resistance rates faced by fluoroquinolones. This observation permits emphasize that population variability may play an important role on the selecting process of antibiotics for prophylaxis purposes. Consequently, knowledge of the bacterial resistance profile from the local population is paramount for optimizing post-biopsy infectious complications results. However, this study results have limitations, a control group was not designed for comparisons and therefore its evidence level was reduced to grade III. Other aspect is that post-biopsy urine culture was positive in four asymptomatic patients and was not considered as an infection event. Asymptomatic bacteriuria can lead to oligosymptomatic urine/prostate colonization causing eventual urinary tract infection onset in the future.

Is the era of empiric fluoroquinolones for prostate biopsy prophylaxis over?

Prostate biopsy is the gold-standard method for diagnosing prostate cancer. The procedure is most commonly performed through a transrectal approach, which can expose the genital and urinary tract to Gram-negative enterobacteria infection, especially caused by E. coli (1).

A Cochrane review on prophylaxis for transrectal prostate biopsy revealed a significant reduction in bacteriuria, urinary tract infection, bacteremia, fever and hospitalization after prostate biopsy with antibiotics compared to placebo. Definitive evidences of superiority of long-term or multiple-dose compared to short-term or single-dose antibiotic prophylaxis protocols were not demonstrated (2). Several reports have not shown significant difference between single-dose/1-day and 3-day prophylactic regimens (3-5). In addition, the American Urological Association has recommended antibiotic prophylaxis maintained for less than 24 hours in transrectal prostate biopsies (6). In this scenario, a short-term protocol may offer advantages of cost savings with potentially fewer drug related resistance.

Other efforts for reducing post-biopsy infection rates have been investigated. The use of enemas in association with antibiotic prophylaxis was also evaluated by Cochrane review. A reduced risk of bacteremia was identified when this association was applied compared to antibiotics alone, although no differences were found in fever or infection endpoints (2).

Patient-specific and procedure-specific characteristics were also described as possible potential risk factors for higher post-biopsy infection rates: increased comorbidity scores, untreated asymptomatic bacteriuria, history of prostatitis, urinary tract infection, prostate size, indwelling urinary catheters, presence of bladder stones, inadequately treated diabetes mellitus, number of biopsy cores and number of repeat biopsy procedures (7).

The choice of the prophylactic antibiotic type has been empirical and guided by the expected bacterial spectrum at the operative site, antibiotic susceptibility, drug pharmacokinetic and pharmacodynamics properties (8). Fluoroquinolones have traditionally been used as the primary prophylactic agent for prostate biopsy due to excellent urinary and prostatic penetration providing optimal coverage against key pathogens (9).

Despite the fact transrectal prostate biopsy has been widely considered a safe procedure for a long period of time and associated with low infectious complications rates, contemporary prospective and retrospective reports have currently shown a surprisingly increase on post-biopsy infectious rates from 1\% to $4 \%$ over the past fifteen years $(10,11)$. Parallel to this trend, studies have also shown a dramatically increase in the prevalence of fluoroquinolone-resistant E. coli strains (12). These findings have progressively changed the optimal scenario found by fluoroquinolones to effectively promote antibiotic prophylaxis in transrectal prostate biopsies.

Another important issue is that the previous controlled randomized trials that first evaluated the empirical use of antibiotic prophylaxis before transrectal prostate biopsies were performed when levels of resistance to commonly used antibiotics were generally low (2). 
In this respect, recent reports demonstrated presence of fluoroquinolone-resistant bacteria in 50 - 90\% of patients with post-biopsy symptomatic infections. Additionally, the presence of fluoroquinolone-resistant pathogens in the rectal flora preoperatively, has been considered the most important risk factor for post-biopsy infection. The risk of harboring fluoroquinolone-resistant bacteria in the faeces was evaluated and increased remarkably on those who have received fluoroquinolones within the past 6 months or after international travel to countries with high levels of antibiotic resistance (12-14).

In this regard, non-randomized trials applied rectal swab cultures for preoperative assessment of rectal flora susceptibility and performed a targeted antimicrobial therapy based on its susceptibility profile. The targeted antibiotic prophylaxis was associated with a notable decrease in the incidence of infectious complications as well as a decrease in the overall cost of care (15).

Currently, these new attempts for a more individualized and optimized antibiotic prophylaxis based on the susceptibility profile of the rectal flora of each patient reached a new milestone of a new era on the way for reducing post biopsies infection rates. However, additional larger randomized prospective studies are still needed to further evaluate the efficacy and cost-effectiveness of this new strategy and compare it to the traditional empirical prophylaxis approach.

On the other hand, new biopsy technology as the MRI-transrectal ultrasonography (MRI-TRUS) fusion-guided-3D targeted biopsies has potential to reduce the number of repeated biopsies $(16,17)$. Consequently, it may reduce the amount of antibiotic used for prophylaxis and therefore possibly contribute for reducing antibiotic resistance in the future.

\section{REFERENCES}

1. Tobias-Machado M, Verotti MJ, Aragao AJ, Rodrigues AO, Borrelli M, Wroclawski ER: Prospective randomized controlled trial comparing three different ways of anesthesia in transrectal ultrasound-guided prostate biopsy. Int Braz J Urol. 2006; 32: 172-9; discussion 179-80.
2. Zani EL, Clark OA, Rodrigues Netto N Jr.: Antibiotic prophylaxis for transrectal prostate biopsy. Cochrane Database Syst Rev. 2011; 5: CD006576.

3. Shigemura K, Tanaka K, Yasuda M, Ishihara S, Muratani T, Deguchi $T$, et al.: Efficacy of 1-day prophylaxis medication with fluoroquinolone for prostate biopsy. World J Urol. 2005; 23: 356-60.

4. Tobias-Machado M, Corrêa TD, De Barros EL, Wroclawski ER: Antibiotic prophylaxis in prostate biopsy. A comparative randomized clinical assay between ciprofloxacin, norfloxacin and chloramphenicol. Int Braz J Urol. 2003; 29: 313-9.

5. Aron M, Rajeev TP, Gupta NP: Antibiotic prophylaxis for transrectal needle biopsy of the prostate: a randomized controlled study. BJU Int. 2000; 85: 682-5.

6. Wolf JS Jr, Bennett CJ, Dmochowski RR, Hollenbeck BK, Pearle MS, Schaeffer AJ; et al.: Best practice policy statement on urologic surgery antimicrobial prophylaxis. J Urol. 2008; 179: 1379-90. Erratum in: J Urol. 2008; 180: 2262-3.

7. Loeb S, Vellekoop A, Ahmed HU, Catto J, Emberton M, Nam $\mathrm{R}$, Rosario DJ, et al.: Systematic review of complications of prostate biopsy. Eur Urol. 2013; 64: 876-92.

8. Wagenlehner FM, Grabe M, Naber KG, Bjerklund Johansen TE, Naber CK, Weidner W: [Antibiotic prophylaxis in urology]. Urologe A. 2011; 50: 1469-78; quiz 1479-80.

9. Al-Hasan MN, Lahr BD, Eckel-Passow JE, Baddour LM: Antimicrobial resistance trends of Escherichia coli bloodstream isolates: a population-based study, 1998-2007. J Antimicrob Chemother. 2009; 64: 169-74.

10. Loeb S, Carter HB, Berndt SI, Ricker W, Schaeffer EM: Complications after prostate biopsy: data from SEERMedicare. J Urol. 2011; 186: 1830-4.

11. Loeb S, van den Heuvel S, Zhu X, Bangma CH, Schröder $\mathrm{FH}$, Roobol MJ: Infectious complications and hospital admissions after prostate biopsy in a European randomized trial. Eur Urol. 2012; 61: 1110-4.

12. Williamson DA, Roberts SA, Paterson DL, Sidjabat $H$, Silvey A, Masters J, et al.: Escherichia coli bloodstream infection after transrectal ultrasound-guided prostate biopsy: implications of fluoroquinolone-resistant sequence type 131 as a major causative pathogen. Clin Infect Dis. 2012; 54: 1406-12.

13. Patel U, Dasgupta P, Amoroso P, Challacombe B, Pilcher $\mathrm{J}$, Kirby R: Infection after transrectal ultrasonographyguided prostate biopsy: increased relative risks after recent international travel or antibiotic use. BJU Int. 2012; 109: 1781-5.

14. Williamson DA, Masters J, Freeman J, Roberts S: Travelassociated extended-spectrum $\beta$-lactamase-producing Escherichia coli bloodstream infection following transrectal ultrasound-guided prostate biopsy. BJU Int. 2012; 109: E21-2. 
15. Taylor AK, Zembower TR, Nadler RB, Scheetz MH, Cashy JP, Bowen D, et al.: Targeted antimicrobial prophylaxis using rectal swab cultures in men undergoing transrectal ultrasound guided prostate biopsy is associated with reduced incidence of postoperative infectious complications and cost of care. J Urol. 2012; 187: 1275-9.

16. Mouraviev V, Verma S, Kalyanaraman B, Zhai QJ, Gaitonde $\mathrm{K}$, Pugnale $\mathrm{M}$, et al.: The feasibility of multiparametric magnetic resonance imaging for targeted biopsy using novel navigation systems to detect early stage prostate cancer: the preliminary experience. J Endourol. 2013; 27: 820-5.
17. Sonn GA, Natarajan $S$, Margolis DJ, MacAiran M, Lieu $P$ Huang $\mathrm{J}$, et al.: Targeted biopsy in the detection of prostate cancer using an office based magnetic resonance ultrasound fusion device. J Urol. 2013; 189: 86-91.

Igor Nunes-Silva, MD and Marcos Tobias-Machado, MD

Section of Urology ABC Medical School Sao Paulo, SP, Brazil E-mail: tobias-machado@uol.com.br 